\title{
CIBERACOSADORES: UN ESTUDIO CUANTITATIVO CON ESTUDIANTES DE SECUNDARIA
}

\section{CYBERBULLIES: A QUANTITATIVE RESEARCH WITH SECONDARY STUDENTS}

\author{
Dr. Víctor González Calatayud \\ victor.gonzalez@um.es \\ Dra. María Paz Prendes Espinosa \\ pazprend@um.es
}

\begin{abstract}
Universidad de Murcia, Facultad de Educación, Departamento de Didáctica y Organización Escolar. Campus Universitario de Espinardo, Facultad de Educación, C.P. 30100, Espinardo (Murcia).
\end{abstract}

\begin{abstract}
Resumen: Uno de los problemas que actualmente más preocupan en los centros de Secundaria es el del acoso escolar y el ciberacoso. Este estudio cuantitativo tiene como objetivo conocer la situación actual del ciberacoso en centros de Secundaria de la Región de Murcia. Con un muestreo por conglomerados, se concretó una muestra representativa que finalmente estuvo compuesta por 950 estudiantes de Educación Secundaria Obligatoria. En este artículo vamos a profundizar en los aspectos relacionados con los ciberacosadores, su prevalencia y el uso que hacen de las tecnologías. Los resultados muestran que un $6.4 \%$ de los participantes se pueden considerar ciberacosadores, aunque un $23.3 \%$ ha realizado alguna de las conductas propias de los ciberacosadores. Además, se corrobora una conclusión obtenida en estudios previos sobre la importancia del control parental. Es importante conocer en profundidad estas conductas para poder diseñar estrategias de prevención en los centros escolares.
\end{abstract}

Palabras clave: TIC; Enseñanza secundaria; Ciberacoso

Abstract: Bullying and cyberbullying are current problems in Secondary Education about which people are worried. The aim of this quantitative research is to know the situation of cyberbullying in the Region of Murcia. The representative sample consisted of 950 students of compulsory secondary education. In this article we are going to analyse some aspects related to cyberbullies, their prevalence and the uses of some technologies that can be risk factors for this problem. The results show that $6.4 \%$ of the participants can be considered as cyberbullies, although $23.3 \%$ have committed one or more of this type of behaviours. Moreover, our study confirms the relevance of parents control to prevent ciberbullying, as other researches have previously founded. It is relevant the knowledge of these profiles to design strategies in order to prevent these situations in scholar institutions.

Keywords: ICT; Secondary Education; Cyberbullying.

Píxel-Bit. Revista de Medios y Educación. No 53 Julio 2018. ISSN: 1133-8482. e-ISSN: 2171-7966. doi: http://dx.doi.org/10.12795/pixelbit.2018.i53.09 


\section{Introducción.}

El problema conocido como «bullying» o acoso tradicional ha sido objeto de numerosos estudios en las últimas décadas, lo que pone de manifiesto la importancia del tema en el mundo científico. Sin embargo, hoy en día hay que sumarle a este problema una nueva variable: las Tecnologías de la Información y la Comunicación (TIC), que han dado lugar a lo que se conoce como ciberacoso o «ciberbullying». Esta problemática ha suscitado gran interés por parte de la comunidad educativa en general y de los docentes en particular (González, Prendes y López, 2016).

Como recogen Garaigordobil y Aliri (2013), el ciberacoso puede ser entendido como el uso que se hace de las TIC, principalmente Internet (correo electrónico, redes sociales, sitios web, blogs, etc.) y el teléfono móvil para realizar un acoso psicológico entre iguales. Además, como Smith et al. (2008) establecen, se trata de un comportamiento agresivo e intencional, realizado por un individuo o un grupo y donde la víctima tiene dificultad para defenderse.

\subsection{El ciberacoso y la tecnología}

Los adolescentes actuales ya han nacido en un mundo donde las TIC tienen una presencia generalizada y han adaptado estas tecnologías a sus quehaceres diarios de una forma casi mecánica (Amichai-Hamburger y Barak, 2009; López y Solano, 2011; Solano, González y López, 2013). En la actualidad, los medios de comunicación e Internet juegan un papel muy significativo en los procesos de socialización y se convierten en medios trascendentales en el desarrollo de la persona y en la construcción de su identidad (Mishna,
Saini y Solomon, 2009; Rubio, 2008). Asimismo, la comunicación a través de la red se rige por sus propias normas y sus usuarios pueden, en ocasiones, interactuar con otras personas de forma muy distinta a las aprendidas en los procesos de socialización que han tenido lugar en espacios físicos (Green, Wilhelmsen, Wilmots, Dodd y Quinn, 2016; Ontiveros, 2015). De igual modo, el mundo virtual también ofrece oportunidades para satisfacer las necesidades de expresión, exploración y experimentación de la propia identidad del sujeto, así como desarrollar nuevos aprendizajes (Chisholm, 2006).

Pero la incorporación de las TIC tiene otra característica muy importante cuando hacemos mención al ciberacoso y es el efecto de desinhibición (Casale, Fiovaranti y Caplan, 2015; Chisholm, 2006; Lapidot-Lefler y Barak, 2012; Suler, 2004; Udris, 2014; Willard, 2004). De esa manera los sujetos se comportarían de forma más abierta, menos restrictiva y más relajada en las relaciones en el mundo virtual. La comunicación en red puede conllevar dos tipos de desinhibición: por un lado, la que promueve la amabilidad y generosidad, llamada benigna; y, por otro lado, otra que lleva consigo un lenguaje más grosero, odio y amenazas, llamada tóxica (Suler, 2004). Este hecho, sumado a que los jóvenes -como indica Boyd (2008)- se comportan de manera distinta a los adultos en la red, y además sumado al anonimato que proporciona la red, podrían dar origen a propiciar situaciones de ciberacoso (Lapidot-Lefler y Barak, 2012; Sticca y Perren, 2013). Además, con la tecnología el tiempo y el espacio se ven afectados (Martínez y Solano, 2003), pudiendo sufrir o realizar ciberacoso en cualquier momento o lugar sin identificar desde dónde o por parte de quiénes. 
Debido a estas particularidades que la tecnología nos ofrece a la hora de comunicarnos y a la facilidad que encuentran los jóvenes para acosar y/o ser acosados, los programas de prevención del ciberacoso suelen recomendar que el ordenador se utilice en un lugar común de la casa, de tal forma que se pueda controlar lo que hacen, ya que lo consideran un factor protector del ciberacoso y de otros riesgos asociados al uso de la red (Bringué y Sádaba, 2011; Luengo Latorre, 2014; Orjuela, Cabrera, Calmaestra, Mora-Merchán y Ortega, 2013). Como se ha concluido en varios estudios (Cerezo, Arnaiz, Gimenez y Maquilón, 2016; Lee y Shin, 2017; Mishna, Khoury-Kassabri, Gadalla, y Daciuk, 2012; Mishna et al., 2009; Twyman, Saylor, Taylor y Comeaux, 2010), el riesgo de estar involucrado en casos de ciberacoso aumenta cuanto mayor sea el acceso a la tecnología en general y a Internet en particular, especialmente en relación a los acosadores (Festl, 2016). Principalmente aquellos que se conectan fuera de casa, lo cual puede ser debido a la falta de supervisión parental, lo que daría lugar a un incremento en el efecto de desinhibición (Udris, 2014). Esta supervisión parental se vuelve un factor clave, ya que como han determinado varias investigaciones (Floros et al., 2013; Ho, Chen y Ng, 2017), con un mayor control se produce un menor riesgo de ciberacoso.

\subsection{Los ciberacosadores}

Al igual que sucede en el acoso tradicional o bullying, varios son los roles de implicación dentro del fenómeno del ciberacoso: cibervíctima, ciberagresor, ciberobservador y cibervíctima-agresiva (Kubiszewski, Fontaine, Potard y Auzoult, 2015). Aunque presentan algunas características diferentes a los roles del acoso tradicional, en cierta manera los roles se asemejan ya que ambos fenómenos están muy relacionados.

En el caso que nos ocupa, el de los ciberacosadores o ciberagresores, aunque la mayoría de estudios se han centrado en investigar a las víctimas de ciberacoso (Schenk, Fremouw, y Keelan, 2013, González, en prensa), son varios los estudios que han investigado distintos aspectos relacionados con los ciberacosadores. Se ha encontrado una gran relación entre los ciberagresores con las agresiones proactivas y reactivas (Calvete, Orue, Estévez, Villardón y Padilla, 2010). Por otro lado, los ciberacosadores tienden igualmente a realizar comportamientos ilegales de forma presencial (Ybarra y Mitchell, 2004), a tener problemas en los centros educativos, agredir a otros físicamente y consumir sustancias ilegales (Hinduja y Patchin, 2008). Por su parte, Schenk et al. (2013) encontraron que los ciberacosadores tuvieron una mayor puntuación en relación con síntomas depresivos, paranoia, psicoticismo y hostilidad.

Aparte de estos aspectos, la prevalencia ha sido de lo más investigado hasta el momento y las cifras halladas son muy dispares. Las prevalencias obtenidas a nivel internacional en estudios previos van desde el 2.9\% (Lam, Cheng y Liu, 2013) hasta el 49.2\% (Yang, Lin y Chen, 2014). No obstante, varios de los que analizan este aspecto se encuentran entre la franja 15-25\% (Estévez, Villardón, Calvete, Padilla y Orue, 2010; Garaigordobil y Aliri, 2013; Huang y Chou, 2010; Patchin y Hinduja, 2010; Pelfrey y Weber, 2013; Walrave y Heirman, 2011). Otro aspecto interesante que nos encontramos es que ciertos estudios encuentran un porcentaje mayor de ciberacosadores que de 
cibervíctimas (Heirman y Walrave, 2012; Müller, Pfetsch y Ittel, 2014; Pelfrey y Weber, 2013; Wong, Chan y Cheng, 2014), siendo el caso de Yang et al. (2014) donde más diferencia se encontró. Uno de los factores que claramente afecta a que existan estas diferencias es el instrumento utilizado (Berne et al., 2013).

\section{Método.}

Este artículo recoge una parte de la investigación cuantitativa realizada en la Región de Murcia en centros de Enseñanza Secundaria Obligatoria en 2016. En concreto, recogemos los datos más significativos obtenidos en relación a los ciberacosadores en un estudio cuantitativo descriptivo de tipo encuesta.

\subsection{Objetivos de la investigación}

Aunque la encuesta ha sido más amplia, en este artículo recogemos los datos directamente relacionados con el objetivo de investigación de identificar la prevalencia de ciberacosadores e identificar el tipo de uso que hacen de las tecnologías para llevar a cabo estos comportamientos de acoso a sus iguales a través de estas herramientas.

\subsection{Diseño}

Es una investigación de tipo descriptivo (ex post facto y transversal) con una metodología cuantitativa y con una técnica de encuesta. Se ha realizado con muestra representativa de estudiantes a los cuales se

\begin{tabular}{|c|c|c|c|c|}
\hline & Nunca & $\begin{array}{l}\text { Algunas } \\
\text { veces }\end{array}$ & $\begin{array}{l}\text { Bastantes } \\
\text { veces }\end{array}$ & Siempre \\
\hline & n (\%) & n (\%) & n (\%) & n (\%) \\
\hline $\begin{array}{l}\text { 1. Mensajes ofensivos o insultantes mediante el móvil o } \\
\text { Internet }\end{array}$ & $831(87.5)$ & $110(11.6)$ & $6(0.6)$ & $3(0.3)$ \\
\hline $\begin{array}{l}\text { 2. Llamadas ofensivas e insultantes mediante el móvil o } \\
\text { Internet }\end{array}$ & $895(94.2)$ & $50(5.3)$ & $4(0.4)$ & $1(0.1)$ \\
\hline $\begin{array}{l}\text { 4. Difundido fotos o vídeos privados de alguien a través } \\
\text { del móvil o de Internet }\end{array}$ & $916(96.4)$ & $30(3.2)$ & $2(0.2)$ & $2(0.2)$ \\
\hline $\begin{array}{l}\text { 6. Llamadas anónimas con el fín de asustar y provocar } \\
\text { miedo }\end{array}$ & $862(90.7)$ & $77(8.1)$ & $8(0.8)$ & $3(0.3)$ \\
\hline $\begin{array}{l}\text { 7. Chantajeado o amenazado por medio de llamadas o } \\
\text { mensajes }\end{array}$ & $916(96.4)$ & $30(3.2)$ & $4(0.4)$ & $0(0)$ \\
\hline $\begin{array}{l}\text { 15. Difamado a alguien por Internet diciendo cosas de esa } \\
\text { persona que son mentira para desprestigiarla }\end{array}$ & $932(98.2)$ & $14(1.5)$ & $3(0.3)$ & $0(0)$ \\
\hline
\end{tabular}

Tabla 1. Frecuencia y porcentaje de conductas de ciberagresión en el último año $-140-$ 
ha aplicado un cuestionario como procedimiento de recogida de información.

\subsection{Participantes}

A partir de un nivel de confianza del $95 \%$ $(Z=1.96)$ y un error muestral del $3,2 \%$, se realizó un muestreo aleatorio por conglomerado. Se obtuvo un tamaño mínimo de 925 casos, los cuales se distribuyeron entre los distritos utilizados por el Servicio de Inspección de la Consejería de Educación de Murcia. La muestra final estuvo compuesta por 950 estudiantes de Educación Secundaria Obligatoria con edades comprendidas entre los 11 y los 18 años, con una media de edad de 13,93 $(\mathrm{DT}=1,35)$, pertenecientes a 10 escuelas públicas $(64.8 \%)$ y concertadas (35.4\%) de la Región de Murcia. La muestra estaba compuesta por $50.4 \%$ de varones y $49.6 \%$ de mujeres. La muestra participante pertenecía a cuatro cursos de Educación Secundaria Obligatoria (ESO) de la siguiente manera: $1^{\circ} \mathrm{ESO}, 28.9 \% ; 2^{\circ} \mathrm{ESO}, 22.9 \% ; 3^{\circ} \mathrm{ESO}$, $23.4 \%$; y $4^{\circ} \mathrm{ESO}, 24.8 \%$. Un $2.7 \%$ informó de algún tipo de discapacidad.

En términos de acceso a las TIC por parte de los participantes de este estudio, el 93.4\% indicó tener un teléfono móvil y un $88.5 \%$ un ordenador personal conectado a Internet. En muchos casos (42\%) el ordenador estaba en el salón, pero el 36.3\% indicó que estaba en su propia habitación. Por último, la mayoría $(83.5 \%)$ indicó que tenía acceso a Internet fuera de casa, encontrando que el teléfono móvil $(71.8 \%)$ es la tecnología más utilizada para acceder a Internet.

\subsection{Instrumento}

Después de realizar una revisión sistemática de los distintos cuestionarios existentes en investigaciones previas sobre el tema se decidió utilizar el cuestionario diseñado por Garaigordobil (2013) titulado «Cyberbullying: Screening of peer harassment». Este cuestionario tiene una fiabilidad y validez estadística muy alta. En nuestra investigación se mostró una fiabilidad global de $\alpha=0,91$ y una muy buena fiabilidad para las subescalas. El análisis factorial exploratorio confirmó la estructura de tres factores dentro de la escala de acoso $(57,89 \%$ de la varianza explicada) y dentro de la escala de ciberacoso $(40,15 \%$ de la varianza explicada). Cada uno de los factores comprende 15 ítems que se refieren a comportamientos diferentes relacionados con los tres roles -víctima, agresor y observadoren el ciberacoso. También mostró una buena validez convergente y divergente.

\subsection{Procedimiento}

En primer lugar, se firmó una hoja de compromiso con la Consejería de Educación de la Región de Murcia. La Consejería se puso en contacto con los centros, que fueron seleccionados al azar. Una vez que los centros habían aceptado participar en la investigación, se les envió una hoja de autorización para que los padres la firmaran y permitieran la participación de sus hijos. El cuestionario fue aplicado después de que todas las autorizaciones firmadas fueran recogidas. Al comienzo de la sesión se explicaba todo el procedimiento a los estudiantes antes de cumplimentar el cuestionario en papel (en total, se empleó un tiempo de entre 15 y 30 minutos con cada grupo de alumnos). 


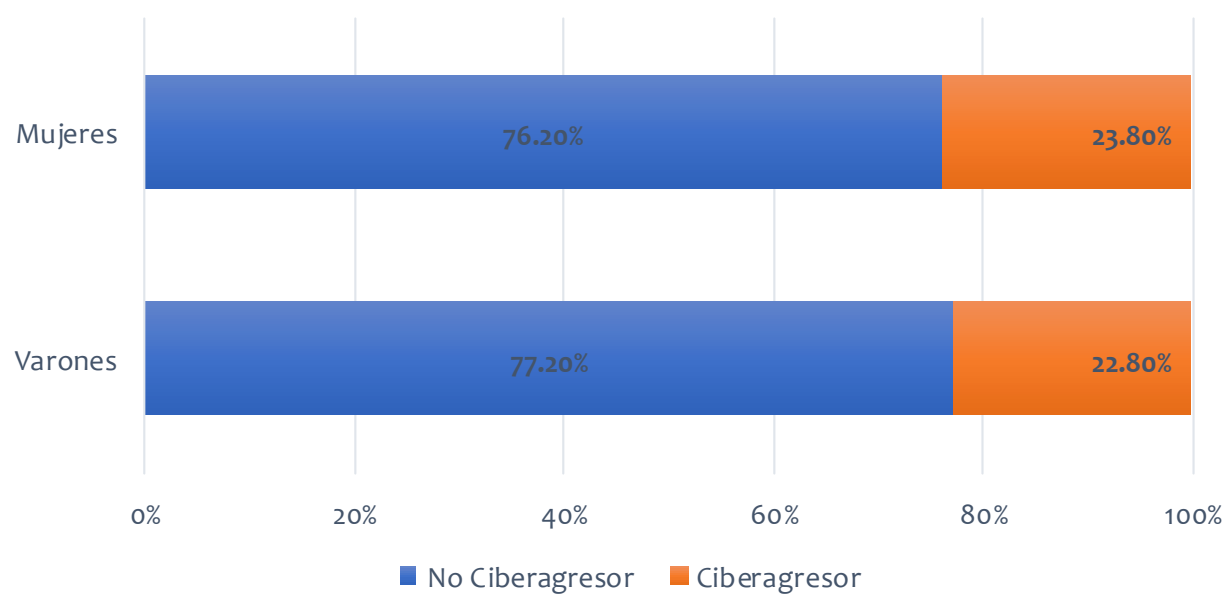

Figura 1. Porcentaje de varones y mujeres ciberagresores

\subsection{Análisis de datos}

Se utilizó el programa estadístico SPSS, versión 22.0, adoptando el nivel de significación bilateral de $\mathrm{p}<0,05$ en todo el estudio. En primer lugar, se realizó un análisis descriptivo de las frecuencias. Para comparar dichas frecuencias, se realizaron tablas de contingencia y se usó el estadístico Chicuadrado. Finalmente, se utilizó la prueba $\mathrm{t}$ de Student y ANOVA para las diferencias en las escalas totales.

\section{Resultados.}

\subsection{Prevalencia de ciberagresión}

En primer lugar, si nos fijamos en las 15 conductas evaluadas en el rol de ciberagresor la prevalencia obtenida en cada una de ellas oscila entre el $0.5 \%$ y el $12.5 \%$, con relación al último año. En la siguiente tabla 1 se reflejan las frecuencias y porcentajes de las conductas más realizadas por los adolescentes de la muestra.

A la hora de establecer los adolescentes que están en riesgo de ser ciberacosadores, la autora del cuestionario utiliza como punto de corte el percentil 95, del cual se desprende que un $6.4 \%$ de la muestra de participantes estaba realmente en riesgo de ser un ciberacosador. Pero si tenemos en cuenta los datos en general de las conductas realizadas se observa que el $23.3 \%$ de la muestra realizó una o más conductas de las que se preguntaban en el cuestionario el último año.

De igual forma, al analizar las diferencias de varones y mujeres que indicaron haber realizado una o más conductas de ciberagresión no se encontró diferencias significativas, $\stackrel{2}{*}^{2}(1,948)=0.14, p=.709$. Tal es el caso que los porcentajes en ambos fueron muy similares (figura 1).

Igualmente, al ver la diferencia de medias según en el sexo en la escala total de ciberagresores se comprobó que no había diferencias significativas, $t(946)=0.09, p=$ 
.931 , hallando que la media en los varones $(M$ $=0.53, D T=1.42)$ y mujeres $(M=0.52, D T=$ 1.43 ) era muy semejante.

\subsection{Relación de los ciberagresores con la tecnología}

El análisis de algunos aspectos relacionados con la tecnología que usan los ciberacosadores puede ser aspectos clave para buscar factores de riesgo asociados a la misma. Por ello, en este apartado se analiza, en primer lugar, la influencia de la posición del ordenador en la casa de los participantes. De este modo, de aquellos que indicaron no tener un ordenador conectado en casa solamente 20 participantes $(18.3 \%)$ realizó alguna conducta de ciberacoso, mientras que de los que si tenían ordenador 201 (23.9\%) llevó a cabo alguna ciberagresión. Sin embargo, estas diferencias no produjeron significación en la prueba Chi-cuadrado de Pearson, $\stackrel{\circ}{*}^{2}(1,950)=1.67, p=.197$. Además, tampoco se encontraron diferencia significativa en la media de la escala total de ciberagresión entre aquello que si tenían ordenador conectado $(M=0.51, D T=1.30) \mathrm{y}$ aquellos que no $(M=0.61, D T=2.16), t(948)$ $=-0.70, p=.481, d=-.06$.

Por su parte, aquellos que indicaron tener un ordenador conectado en el salón obtienen porcentajes similares en la realización de una o más conductas de ciberacoso $(n=89$, $22.3 \%)$ que aquellos no lo tenían ahí $(n=131$, $23.8 \%)$. Por tanto, no se encontró significación en la prueba Chi-cuadrado, $\div^{2}$ $(1,950)=0.30, p=.586$. No obstante, al analizar la diferencia de la media en la escala total de ciberagresión se comprobó que aquellos que tienen el ordenador en el salón $(M=0.41, D T$ $=0.89$ ) obtuvieron una media inferior que aquellos que no lo tienen ahí $(M=0.61, D T=$ $1.71), t(868.64)=2.38, p<.05$, con un tamaño del efecto bajo $d=.16$.

De igual forma, se halló que de aquellos que tenían el ordenador conectado en su habitación casi un tercio $(n=100,29 \%)$ realizó una o más conductas de ciberacoso, mientras

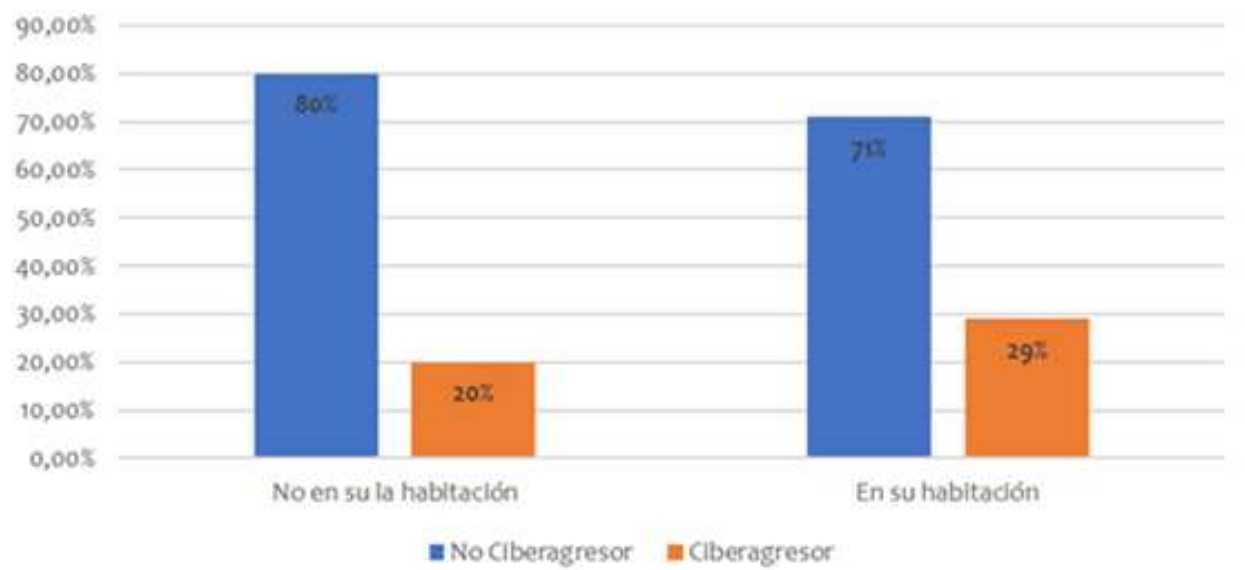

Figura 2. Porcentajes de ciberagresores en relación con tener ordenador conectado en su habitación 


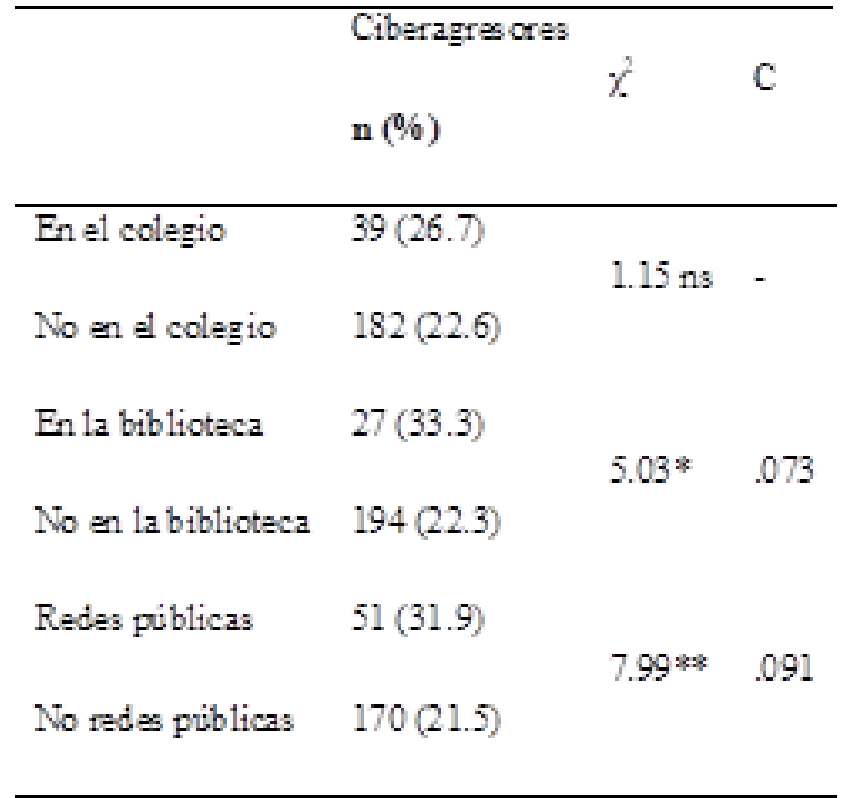

Nota: $* p<.05, * * p<.01, \mathrm{~ns}=$ no significativo, $\mathrm{C}=$ Coeficiente de Contingencia

Tabla 2. Frecuencia, porcentaje y Chi-cuadrado de ciberagresores en lugares de conexión

de los que no tenían en su habitación ( $n=$ $121,20 \%$ ) ese porcentaje baja (figura 2 ). Tales diferencias reflejaron una diferencia significativa con el estadístico Chi-cuadrado de Pearson, $\div \cdot \div(1,950)=9.98, p<.01, C=.102$. Además, aquellos que sí tenían el ordenador en su habitación obtuvieron más media en la escala total de ciberagresión $(M=0.61, D T=$ 1.38) que aquellos que no lo tenían en su habitación $(M=0.47, D T=1.45)$, aunque dicha diferencia no resultó significativa, $t(948)=$ $1.48, p=.140, d=.10$.

En la escala total de ciberagresión se observó que aquellos que se conectan desde fuera de casa a Internet realizan una o más conductas de ciberacoso $(n=198,24.9 \%)$ que aquellos que no se conectan $(n=23,14.8 \%)$, hallando una asociación entre ambas variables, $\dot{\div}^{2}(1,950)=7.36, p<.01, C=.088$.
Igualmente, la media en la escala general de los que no se conecta $(\mathrm{M}=0.21, \mathrm{DT}=0.59)$ es bastante menor que la que sí se conectan fuera de casa $(\mathrm{M}=0.58, \mathrm{DT}=1.53)$, encontrando que dicha diferencia era significativa, $t(612.6)=-5.16, p<.001, d=$ .42 .

Asimismo, dentro de los que indicaron que sí se conectaban fuera de casa, un $86 \%$ ( $n=$ 684) lo hacían a través de un smartphone. Y de todos estos una cuarta parte $(n=177$, $25.9 \%$ ) realizó una o más conductas de ciberacoso, siendo también significativo, $\div^{2}$ $(1,950)=9.19, p<.01, C=.098$. Igualmente, de aquellos que usan una Tablet para conectarse fuera de casa $(n=91,9.6 \%)$ el porcentaje de ciberagresores fue mayor en aquellos que sí la usaban $(n=26,28.6 \%)$ que de los que no la usaron $(n=195,22.7 \%)$, 
aunque no fue significativa la diferencia, $\div$ $(1,950)=1.59, p=.208$. En cuanto al lugar donde se conectan se hallaron diferencias significativas, obteniendo una media mayor aquellos que se conectaban desde los distintos sitios que se preguntaban (tabla 2). Concretamente se hallaron diferencias en esta escala entre aquellos que se conectaron desde redes públicas $(M=0.93, D T=2.17)$ y los que no lo hicieron $(M=0.44, D T=1.20), t$ $(179.37)=2.73 p<.01, d=.41$.

\section{Discusión.}

El número de participantes que indicaron haber realizado una o más conductas de ciberacoso hacia otros en el último año alcanza a un 23,3\%, lo cual es una cifra bastante considerable. Es más, este porcentaje de ciberacosadores es similar al encontrado en otros estudios que obtuvieron porcentajes entre el $15 \mathrm{y}$ el $25 \%$ (Calvete et al., 2010; Garaigordobil y Aliri, 2013; Huang y Chou, 2010; Patchin y Hinduja, 2010; Pelfrey y Weber, 2013; Walrave y Heirman, 2011), aunque es algo inferior al encontrado por Wong et al. (2014) y Yang et al. (2014). Si tenemos en cuenta el punto de corte establecido por la autora del instrumento, el porcentaje de adolescentes que se pueden considerar en riesgo de ser ciberacosadores alcanza al 6,4\%. Este porcentaje es superior al estudio realizado por Garaigordobil (2013), en el que se utilizó el mismo instrumento, donde obtuvieron un porcentaje de $4,8 \%$. Como los datos indican, no existen diferencias de género en el número de ciberacosadores, siendo los porcentajes muy similares. Este dato se contrapone al encontrado en el número de víctimas (González, en prensa), en el que las mujeres fueron más cibervíctimas que los varones.
En cuanto a las 15 conductas evaluadas, los resultados indicaron que entre el $0.5 \%$ y el $12.5 \%$ había realizado alguna de las mismas, incluso en algunos casos esas acciones se realizaban de forma frecuente por los participantes. Destacan como las conductas que más realizaron los estudiantes el envío de mensajes ofensivos a través del móvil e Internet, la realización de llamadas anónimas para asustar y hacer llamadas ofensivas e insultantes a través del móvil o Internet. Estas conductas coinciden con el estudio de Garaigordobil (2015), aunque los porcentajes son algo superiores.

Si nos centramos en el uso de la tecnología, presumiblemente, el acceso a la misma coloca a los menores en un mayor riesgo de estar involucrados en situaciones de ciberacoso, ya que son el medio de expresión de este problema. Además, no se puede eliminar que los jóvenes consideran que las TIC son el medio dominante para comunicarse con sus compañeros (Mishna et al., 2009). Por lo tanto, uno de los objetivos es investigar la relación con algunas tecnologías utilizadas por los adolescentes, siendo uno de los principales aspectos a investigar la ubicación del ordenador en la casa. En muchos programas de prevención se considera un factor de riesgo el hecho de ubicar el ordenador en la habitación del menor, tanto para ser víctima como para ser acosador, por lo que se suele recomendar ubicar el ordenador en un lugar común (Bringué y Sádaba, 2011; Luengo Latorre, 2014; Orjuela et al., 2013).

A través de los resultados se puede concluir que el uso de Internet fuera de casa -tanto con ordenadores como con teléfonosestá asociado con una mayor participación en general en los casos de ciberacoso, dato que coincide con las conclusiones de otros autores (Cerezo et al., 2016; Lee y Shin, 2017; 
Mishna et al., 2012, 2009; Twyman et al., 2010), sobre todo si la conducta en cuestión se puede ver por una mayor audiencia (Festl, 2016). Especialmente, aquellos que se conectan a Internet fuera de la casa y utilizan las redes inalámbricas públicas realizan más conductas de intimidación a través de la tecnología. El acceso a Internet fuera de casa implica una falta de supervisión que puede incrementar el efecto de desinhibición que ya de por sí tienen las TIC y, por lo tanto, incrementar el ciberacoso (Udris, 2014).

Además, se puede concluir a partir de los datos que el uso de un ordenador conectado a Internet en la habitación propia del adolescente es un factor de riesgo para la realización de conductas relacionadas con el ciberacoso. Por lo tanto, es conveniente que los programas de prevención recomienden a los padres la colocación del ordenador en un área común de la casa para facilitar el control parental. Relacionado con ello, encontramos varios estudios que han vinculado el estilo parental de crianza con el ciberacoso, demostrando que un mayor control en lo que hacen los adolescentes por parte de los padres se asocia con una menor participación en los casos de ciberacoso (Floros et al., 2013; Ho et al., 2017).

A la vista de todos estos datos, podemos concluir que es necesario un mayor control por parte de los padres en lo que hacen sus hijos con la tecnología. En algunos casos será necesario tener el ordenador ubicado en un sitio común. En otras situaciones se requerirá algún tipo de acuerdo entre el menor y los padres para que se pueda tener un cierto control de lo que hacen con el móvil o la tableta fuera de casa. De esta manera podremos evitar no solo que nuestros hijos realicen alguna conducta relacionada con el ciberacoso, sino también evitar que se conviertan en víctimas de esta problemática. Así, se insta a la comunidad educativa a formar también a los padres ante estas nuevas situaciones, ya que es una realidad que no se puede obviar.

Por último, a pesar de los datos destacables de este estudio, existen una serie de limitaciones que pueden dar pie a futuras investigaciones. A pesar de la representatividad de la muestra para la Región de Murcia, los datos no son extrapolables a otras comunidades. Además, los datos obtenidos no nos permiten establecer claramente una distinción entre conductas casuales y frecuentes. Igualmente, este estudio es de corte transversal, por lo que sería conveniente realizar un estudio longitudinal para comprobar si las conductas se mantienen a lo largo del tiempo.

\section{Fuentes de financiación}

Este trabajo forma parte de un proyecto para conocer la prevalencia del ciberacoso y la percepción de los docentes y ha sido posible gracias a la financiación de la Universidad de Murcia, dentro de su programa de Formación del Profesorado Universitario (FPU).

\section{Referencias bibliográficas}

Amichai-Hamburger, Y., \& Barak, A. (2009). Internet and well-being. In Y. AmichaiHamburger (Ed.), Technology and psychological well-being (pp. 34-76). Cambridge, UK: Cambridge University Press.

Berne, S., Frisén, A., Schultze-Krumbholz, A., Scheithauer, H., Naruskov, K., Luik, P., ... Zukauskiene, R. (2013). Cyberbullying assessment instruments: A systematic review. Aggression and Violent Behavior, 18(2), 320334. doi: 10.1016/j.avb.2012.11.022 
Boyd, D. (2008). Why youth love social network sites: The role of networked publics in teenage life. In D. Buckingham (Ed.), Youth, Identity, and Digital media (pp. 119-143). Cambridge, MA: MIT Press.

Bringué, X., \& Sádaba, C. (2011). Menores y redes sociales. Madrid: Foro Generaciones Interactivas.

Calvete, E., Orue, I., Estévez, A., Villardón, L., \& Padilla, P. (2010). Cyberbullying in adolescents: Modalities and aggressors' profile. Computers in Human Behavior, 26(5), 1128-1135. doi: 10.1016/ j.chb.2010.03.017

Casale, S., Fiovaranti, G., \& Caplan, S. (2015). Online Disinhibition. Journal of Media Psychology, 27(4), 170-177. doi: 10.1027/18641105/a000136

Cerezo, F., Arnaiz, P., Gimenez, A. M., \& Maquilón, J. J. (2016). Conductas de ciberadicción y experiencias de cyberbullying entre adolescentes. Anales de Psicología, 32(3), 761. doi: 10.6018/analesps.32.3.217461

Chisholm, J. F. (2006). Cyberspace Violence against Girls and Adolescent Females. Annals of the New York Academy of Sciences, 1087(1), 74-89. doi: 10.1196/annals.1385.022

Estévez, A., Villardón, L., Calvete, E., Padilla, P., \& Orue, I. (2010). Adolescentes víctimas de cyberbullying: prevalencia y características. Psicothema, 18(1), 73-89.

Festl, R. (2016). Perpetrators on the internet: Analyzing individual and structural explanation factors of cyberbullying in school context. Computers in Human Behavior, 59, 237-248. doi: 10.1016/j.chb.2016.02.017

Floros, G., Paradeisioti, A., Hadjimarcou, M., Mappouras, D. G., Kalakouta, O., Avagianou, P., \& Siomos, K. (2013). Cyberbullying in Cyprus-associated parenting style and psychopathology. Studies in Health
Technology and Informatics, 191, 85-9. doi: 10.3233/978-1-61499-282-0-85

Garaigordobil, M. (2013). Cyberbullying: Screening de acoso entre iguales. Madrid: TEA Ediciones.

Garaigordobil, M. (2015). Ciberbullying en adolescentes y jóvenes del País Vasco: Cambios con la edad. Anales de Psicología, 31(3), 1069-1076. doi: 10.6018/ analesps.31.3.179151

Garaigordobil, M., \& Aliri, J. (2013). Ciberacoso en el País Vasco: Diferencias de sexo en víctimas, agresores y observadores. Psicología Conductual, 21(3), 461-474.

González, V. (en prensa). Victims of cyberbullying in the Region of Murcia: a quantitative study with high school students. NAER, New Approaches in Educational Research

González, V., Prendes, M. P., \& López, J. A. (2016). La percepción sobre el ciberacoso del profesorado de secundaria de la Región de Murcia. Innoeduca. International Journal of Technology and Educational Innovation, 2(2), 84. doi: 10.20548/ innoeduca.2016.v2i2.1974

Green, T., Wilhelmsen, T., Wilmots, E., Dodd, B., \& Quinn, S. (2016). Social anxiety, attributes of online communication and selfdisclosure across private and public Facebook communication. Computers in Human Behavior, 58, 206-213. doi: 10.1016/ j.chb.2015.12.066

Heirman, W., \& Walrave, M. (2012). Predicting adolescent perpetration in cyberbullying: an application of the theory of planned behavior. Psicothema, 24(4), 614620. Recuperado de http:// www.ncbi.nlm.nih.gov/pubmed/23079360

Hinduja, S., \& Patchin, J. W. (2008). Cyberbullying: An Exploratory Analysis of Factors Related to Offending and 
Victimization. Deviant Behavior, 29(2), 129156. doi: 10.1080/01639620701457816

Ho, S. S., Chen, L., \& Ng, A. P. Y. (2017). Comparing cyberbullying perpetration on social media between primary and secondary school students. Computers y Education, 109, 74-84. doi: 10.1016/ j.compedu.2017.02.004

Huang, Y., \& Chou, C. (2010). An analysis of multiple factors of cyberbullying among junior high school students in Taiwan. Computers in Human Behavior, 26(6), 15811590. doi: 10.1016/j.chb.2010.06.005

Kubiszewski, V., Fontaine, R., Potard, C., \& Auzoult, L. (2015). Does cyberbullying overlap with school bullying when taking modality of involvement into account? Computers in Human Behavior, 43, 49-57. doi: 10.1016/j.chb.2014.10.049

Lam, L. T., Cheng, Z., \& Liu, X. (2013). Violent online games exposure and cyberbullying/victimization among adolescents. Cyberpsychology, Behavior and Social Networking, 16(3), 159-65. doi: 10.1089/cyber.2012.0087

Lapidot-Lefler, N., \& Barak, A. (2012). Effects of anonymity, invisibility, and lack of eye-contact on toxic online disinhibition. Computers in Human Behavior, 28(2), 434443. doi: 10.1016/j.chb.2011.10.014

Lee, C., \& Shin, N. (2017). Prevalence of cyberbullying and predictors of cyberbullying perpetration among Korean adolescents. Computers in Human Behavior, 68, 352-358. doi: 10.1016/j.chb.2016.11.047

López, P., \& Solano, I. M. (2011). Interacción social y comunicación entre jóvenes. In F. Martínez y I. M. Solano (Eds.), Comunicación y relaciones sociales de los jóvenes en la red (pp. 27-46). Alcoy: Marfil S.A.

Luengo Latorre, J. A. (2014). Ciberbullying: Prevenir y actuar. Colegio oficial de Psicólogos de Madrid. Recuperado de http://www.copmadrid.org/webcopm/ recursos/CiberbullyingB.pdf

Martínez, F., \& Solano, I. M. (2003). El proceso comunicativo en situaciones virtuales. In F. Martínez (Ed.), Redes de comunicación en la enseñanza: las nuevas perspectivas del trabajo corporativo (pp. 13-29). Barcelona: Ediciones Paidós Ibérica S.A.

Mishna, F., Khoury-Kassabri, M., Gadalla, T., \& Daciuk, J. (2012). Risk factors for involvement in cyber bullying: Victims, bullies and bully-victims. Children and Youth Services Review, 34(1), 63-70. doi: 10.1016/ j.childyouth.2011.08.032

Mishna, F., Saini, M., \& Solomon, S. (2009). Ongoing and online: Children and youth's perceptions of cyber bullying. Children and Youth Services Review, 31(12), 1222-1228. doi: 10.1016/j.childyouth.2009.05.004

Müller, C. R., Pfetsch, J., \& Ittel, A. (2014). Ethical Media Competence as a Protective Factor Against Cyberbullying and Cybervictimization Among German School Students. Cyberpsychology, Behavior, and Social Networking, 17(10), 644-651. doi: 10.1089/cyber.2014.0168

Ontiveros, E. (2015). Treinta años después: Evidencias e interrogantes. Revista TELOS, Cuadernos de Comunicación E Innovación, 100, 1-4.

Orjuela, L., Cabrera, B., Calmaestra, J., MoraMerchán, J. A., \& Ortega, R. (2013). Acoso escolar y ciberacoso/: propuestas para la acción. Save the Children. Recuperado de https://www.savethechildren.es/sites/ $\mathrm{default/file} \mathrm{s/i} \mathrm{m} \mathrm{c} \mathrm{e} \mathrm{/} \mathrm{d} \mathrm{o} \mathrm{c} \mathrm{s} \mathrm{/}$ acoso_escolar_y_ciberacoso_informe_vok__05.14.pdf

Patchin, J. W., \& Hinduja, S. (2010). Cyberbullying and Self-Esteem. Journal of 
School Health, 80(12), 614-621. doi: 10.1111/ j.1746-1561.2010.00548.x

Pelfrey, W. V., \& Weber, N. L. (2013). Keyboard Gangsters: Analysis of Incidence and Correlates of Cyberbullying in a Large Urban Student Population. Deviant Behavior, 34(1), 68-84. doi: 10.1080/ 01639625.2012 .707541

Rubio, Á. (2008). Adolescentes y jóvenes en la red: Factores de oportunidad. Madrid: Instituto de la Juventud, Ministerio de Igualdad.

Schenk, A. M., Fremouw, W. J., \& Keelan, C. M. (2013). Characteristics of college cyberbullies. Computers in Human Behavior, 29(6), 2320-2327. doi: 10.1016/ j.chb.2013.05.013

Smith, P. K., Mahdavi, J., Carvalho, M., Fisher, S., Russell, S., \& Tippett, N. (2008). Cyberbullying: its nature and impact in secondary school pupils. Journal of Child Psychology and Psychiatry, 49(4), 376-385. doi: 10.1111/j.1469-7610.2007.01846.x

Solano, I. M., González, V., \& López, P. (2013). Adolescentes y comunicación: Las TIC como recurso para la interacción social en educación secundaria. Pixel Bit. Revista de Medios Y Educación, (42), 23-35.

Sticca, F., \& Perren, S. (2013). Is cyberbullying worse than traditional bullying? Examining the differential roles of medium, publicity, and anonymity for the perceived severity of bullying. Journal of Youth and Adolescence, 42(5), 739-50. doi: 10.1007/s10964-012-9867-3

Suler, J. (2004). The Online Disinhibition Effect. CyberPsychology y Behavior, 7(3), 321-326. doi: 10.1089/1094931041291295

Twyman, K., Saylor, C., Taylor, L. A., \& Comeaux, C. (2010). Comparing children and adolescents engaged in cyberbullying to matched peers. Cyberpsychology, Behavior and Social Networking, 13(2), 195-9. Recuperado de http://www.ncbi.nlm.nih.gov/ pubmed/20528278

Udris, R. (2014). Cyberbullying among high school students in Japan: Development and validation of the Online Disinhibition Scale. Computers in Human Behavior, 41, 253-261. doi: 10.1016/j.chb.2014.09.036

Walrave, M., \& Heirman, W. (2011). Cyberbullying: Predicting Victimisation and Perpetration. Children y Society, 25(1), 5972. doi: 10.1111/j.1099-0860.2009.00260.x

Willard, N. E. (2004). I Can't See You - You Can't See Me. How the Use of Information and Communication Technologies Can Impact Responsible Behavior. Recuperado de http://goo.gl/EO4WqJ

Wong, D. S. W., Chan, H. C. (Oliver), \& Cheng, C. H. K. (2014). Cyberbullying perpetration and victimization among adolescents in Hong Kong. Children and Youth Services Review, 36, 133-140. doi: 10.1016/j.childyouth.2013.11.006

Yang, S. C., Lin, C., \& Chen, A. (2014). A Study of Taiwanese Teens' Traditional and Cyberbullying Behaviors. Journal of Educational Computing Research, 50(4), 525-552. doi: 10.2190/EC.50.4.e

Ybarra, M. L., \& Mitchell, K. J. (2004). Online aggressor/targets, aggressors, and targets: A comparison of associated youth characteristics. Journal of Child Psychology and Psychiatry and Allied Disciplines, 45(7), 1308-1316. doi: $10.1111 / \mathrm{j} .1469$ 7610.2004.00328.x

Fecha de recepción: 18-07-2017

Fecha de evaluación: 03-09-2017

Fecha de aceptación: 07-09-2017 